\title{
$\beta$-Hydroxybutyric Sodium Salt Inhibition of Growth Hormone and Prolactin Secretion via the cAMP/PKA/CREB and AMPK Signaling Pathways in Dairy Cow Anterior Pituitary Cells
}

\author{
Shou-Peng Fu ${ }^{1,2, \dagger}$, Wei Wang ${ }^{1, \dagger}$, Bing-Run Liu ${ }^{1, \dagger}$, Huan-Min Yang ${ }^{2, \dagger}$, Hong Ji ${ }^{2, \dagger}$, \\ Zhan-Qing Yang ${ }^{1}$, Bin Guo ${ }^{1}$, Ju-Xiong Liu ${ }^{1, *}$ and Jian-Fa Wang ${ }^{1,2, *}$ \\ 1 College of Veterinary Medicine, Jilin University, Changchun 130062, China; \\ E-Mails: shoupengfu@163.com (S.-P.F.); wang_wei99@jlu.edu.cn (W.W.); \\ lhglbr@163.com (B.-R.L.); yangzhanqing1983@163.com (Z.-Q.Y.); guobin79@jlu.edu.cn (B.G.) \\ 2 College of Animal Science and Veterinary Medicine, Heilongjiang Bayi Agricultural University, \\ Daqing 163319, China; E-Mails: yanghuanmin@aliyun.com (H.-M.Y.); \\ jihonghljbynd@aliyun.com (H.J.)
}

$\dagger$ These authors contributed equally to this work.

* Authors to whom correspondence should be addressed;

E-Mails: juxiongliu@sina.com (J.-X.L.);wjflw@sina.com (J.-F.W.);

Tel./Fax: +86-431-8783-6163 (J.-X.L.).

Academic Editor: Kathleen Van Craenenbroeck

Received: 17 November 2014 / Accepted: 9 February 2015 / Published: 16 February 2015

\begin{abstract}
BHBA) regulates the synthesis and secretion of growth hormone $(\mathrm{GH})$ and prolactin (PRL), but its mechanism is unknown. In this study, we detected the effects of BHBA on the activities of $G$ protein signaling pathways, AMPK- $\alpha$ activity, $G H$, and $P R L$ gene transcription, and GH and PRL secretion in dairy cow anterior pituitary cells (DCAPCs). The results showed that BHBA decreased intracellular cAMP levels and a subsequent reduction in protein kinase A (PKA) activity. Inhibition of PKA activity reduced cAMP response element-binding protein (CREB) phosphorylation, thereby inhibiting GH and PRL transcription and secretion. The effects of BHBA were attenuated by a specific $\mathrm{G}_{\alpha i}$ inhibitor, pertussis toxin (PTX). In addition, intracellular BHBA uptake mediated by monocarboxylate transporter 1 (MCT1) could trigger AMPK signaling and result in the decrease in $G H$ and $P R L$ mRNA translation in DCAPCs cultured under low-glucose and non-glucose condition when compared with the high-glucose group. This
\end{abstract}


study identifies a biochemical mechanism for the regulatory action of BHBA on $G H$ and PRL gene transcription, translation, and secretion in DCAPCs, which may be one of the factors that regulate pituitary function during the transition period in dairy cows.

Keywords: $\beta$-hydroxybutyric acid; dairy cow anterior pituitary cells; growth hormone; prolactin

\section{Introduction}

Ketosis is a metabolic disorder that usually occurs in dairy cattle during the early lactation period when cows experience a state of negative energy balance and low blood glucose concentrations [1]. This disorder is characterized by elevated concentrations of the ketone bodies $\beta$-hydroxybutyrate acid (BHBA), acetoacetate and acetone in blood, urine, and milk. In animals, the pituitary is a critical regulator of a broad range of physiological processes involved in growth, metabolism, reproduction, lactation, and stress [2]. The secretion of growth hormone (GH) and prolactin (PRL) from the pituitary gland is modulated by various metabolic influences; however, in vivo experiments have revealed very large differences in the effect of BHBA on GH and PRL secretion depending on the physiological state of the animal. For example, Meier et al. found that during periods of negative energy balance, the somatotrophic axis responds by increasing plasma GH and decreasing plasma IGF-I levels [3]. Laeger et al. reported that high blood BHBA concentrations inhibit the secretion of GH in humans and rhesus monkeys [4]. Thus, results obtained from the studies that used different background animals are not precise. Therefore, the effect and detailed mechanisms by which BHBA mediates bovine pituitary function remain to be elucidated through in vitro studies.

GPR109A is a seven-transmembrane G protein-coupled receptor (GPR) of the Gai family that is expressed mainly in the white adipocytes and immune cells, such as monocytes and neutrophils of humans and mice [5]. The mRNA and protein for GPR109A were observed in fat, muscle, liver and brain of Holstein steers [6]. Moreover, BHBA has been identified as an endogenous ligand of GPR109A [5]. Previously, we found that short-chain fatty acids could inhibit bovine $G H$ and $P R L$ gene transcription via the cAMP-PKA-CREB signaling pathway through GPR41 and GPR43 activation [7]. Thus, we hypothesize that BHBA may mediate bovine $G H$ and $P R L$ gene transcription via the $\mathrm{G}$ protein signaling pathway. AMP-activated protein kinase (AMPK) has emerged as a key molecular player in energy homeostasis at both the cellular and whole-body levels. The incubation of GT1-7 cells with BHBA in the $5.5 \mathrm{mM}$ glucose medium was found to modulate AMPK- $\alpha$ phosphorylation in GT1-7 cells [8]. Pelletier and Coderre also found that BHBA inhibited the activation of the AMPK/p38 MAPK signaling pathway in cardiomyocytes [9]. Recent studies reported that AMPK plays a role in regulating somatotroph function both in the normal rat pituitary and in the proliferation of pituitary adenomatous cells [10]. Therefore, BHBA may regulate bovine pituitary function through the activation of AMPK- $\alpha$ signaling during period of low blood glucose concentrations.

The aim of the present study was to investigate the effect and mechanisms of BHBA on GH and PRL secretion in dairy cow anterior pituitary cells (DCAPCs). To achieve this aim, the activities of G protein signaling pathways, AMPK- $\alpha$ activity, $G H$ and $P R L$ gene transcription, and GH and PRL secretion were 
all determined. The results of this study could provide new knowledge regarding the potential effects of BHBA on bovine pituitary function.

\section{Results}

\subsection{Effect of BHBA on $m R N A$ Levels of GH, PRL and Pit-1 in DCAPCs}

The mRNA levels of $G H, P R L$, and pituitary-specific transcription factor-1 (Pit-1) showed a decreasing trend in the BHBA-treated groups. The mRNA levels of $G H$ were markedly lower after the 24 h BHBA treatment (Figure 1A; $p<0.01$ ), and the mRNA levels of $P R L$ and Pit- 1 were significantly lower after the $24 \mathrm{~h}$ BHBA treatment (Figure 1A; $p<0.05$ ). The mRNA levels of $G H$ were markedly lower in the $0.1,0.5,1.0,2.5$, and $5.0 \mathrm{mmol} / \mathrm{L}$ BHBA treatment groups after $24 \mathrm{~h}$ (Figure $1 \mathrm{~B} ; p<0.01$ ), the mRNA levels of $P R L$ were significantly lower in the 2.5 and $5.0 \mathrm{mmol} / \mathrm{L}$ BHBA treatment groups after $24 \mathrm{~h}$ (Figure 1B; $p<05$ ), and the mRNA levels of Pit-1 were significantly lower in the 0.1, 0.5, 1.0, 2.5 , and $5.0 \mathrm{mmol} / \mathrm{L}$ BHBA treatment groups after $24 \mathrm{~h}$ (Figure $1 \mathrm{~B} ; p<0.05$ ). The mRNA levels of $G H$ were markedly higher in the PTX + BHBA group than in the BHBA treatment group (Figure 1C; $p<0.01$ ), and the mRNA levels of $P R L$ and Pit- 1 were significantly higher in the PTX + BHBA group than in the BHBA treatment group (Figure $1 \mathrm{C} ; p<0.05$ ).

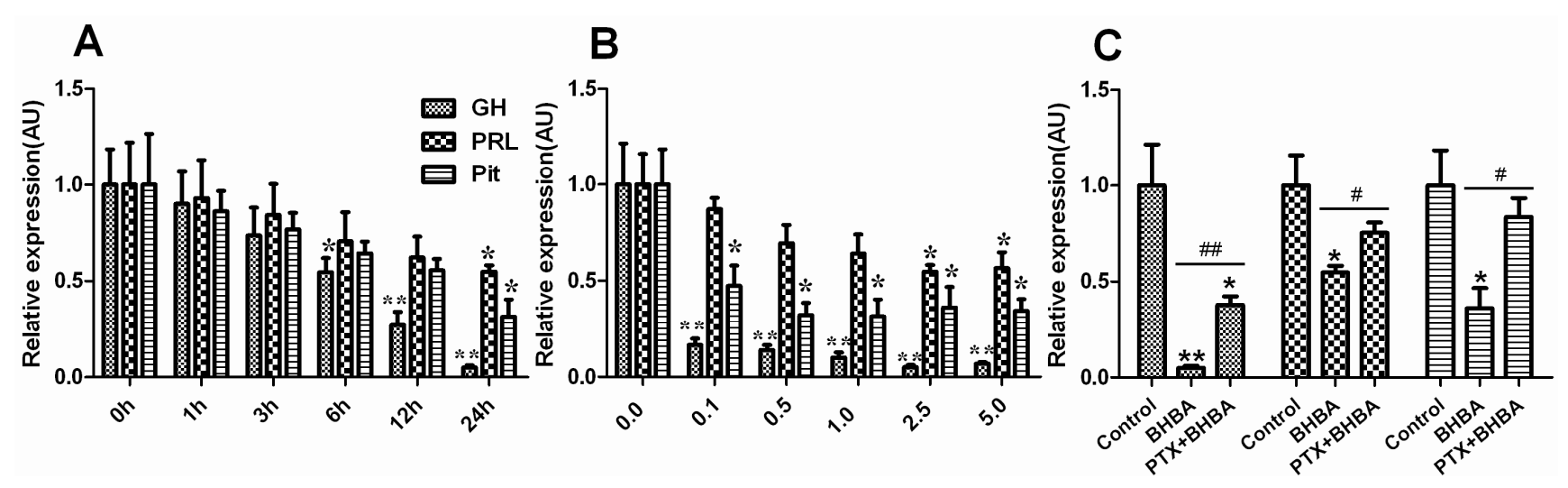

Figure 1. The effect of $\beta$-hydroxybutyric acid (BHBA) on mRNA levels of $G H, P R L$ and Pit-1 in dairy cow anterior pituitary cells (DCAPCs). (A) The effects of the duration of BHBA treatment on GH, PRL, and Pit-1 gene expression; (B) The effects of the dosage of BHBA treatment on the GH, PRL, and Pit-1 gene expression; (C) The results of the mRNA levels of GH, PRL and Pit-1 in DCAPCs treated with or without prior pertussis toxin (PTX) incubation for $2 \mathrm{~h}$ and then stimulated with BHBA for $24 \mathrm{~h}$. * indicates $p<0.05 v s$. the control group, $* *$ indicates $p<0.01$ vs. the control group, ${ }^{\#}$ indicates $p<0.05 v s$. the prior PTX incubation group, ${ }^{\#}$ indicates $p<0.01 v s$. the prior PTX incubation group.

\subsection{Effect of BHBA on GH and PRL Secretion in DCAPCS}

As shown in Figure 2, BHBA notably decreased GH and PRL secretion in DCAPCs in a dose- and time-dependent manner (Figure 2A,B). The secretion levels of GH and PRL were significantly increased in the PTX + BHBA group compared with the BHBA treatment group (Figure $2 \mathrm{C} ; p<0.05$ ). These results indicate that BHBA can decrease GH and PRL transcription and translation in DCAPCs. 

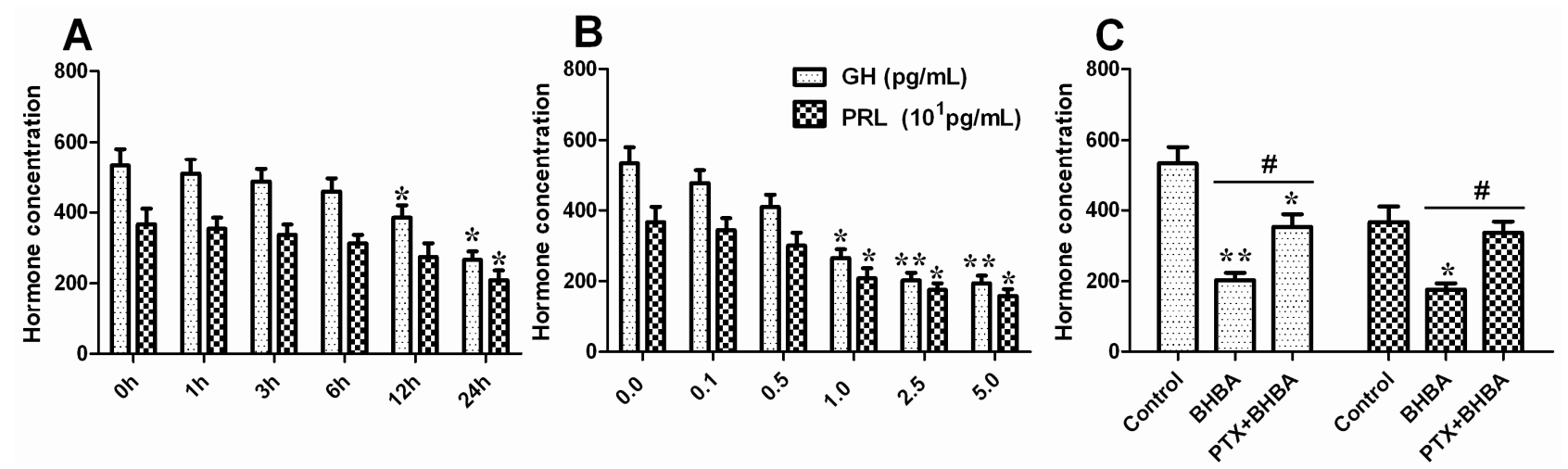

Figure 2. The effect of BHBA on the secretion of GH and PRL in DCAPCs. (A) The effects of the duration of BHBA treatment on GH and PRL secretion; (B) The effects of the dosage of BHBA treatment on GH and PRL secretion; (C) The results of the secretion levels of $\mathrm{GH}$ and PRL in DCAPCs treated with or without prior PTX incubation for $2 \mathrm{~h}$ and then stimulated with BHBA for $24 \mathrm{~h}$. * indicates $p<0.05 v s$. the control group, $* *$ indicates $p<0.01 v s$. the control group, ${ }^{\#}$ indicates $p<0.05 v s$. the prior PTX incubation group.

\subsection{Effect of BHBA on Intracellular cAMP Concentration}

As shown in Figure 3A, BHBA notably decreased the cAMP level in DCAPCs in a time-dependent manner. The cAMP levels were markedly higher in the PTX + BHBA group than in the non-PTX treatment group (Figure 3B; $p<0.01$ ). These results indicate that BHBA can decrease the intracellular cAMP concentration by activating the $\mathrm{G}_{\alpha i}$ subunit in DCAPCs.
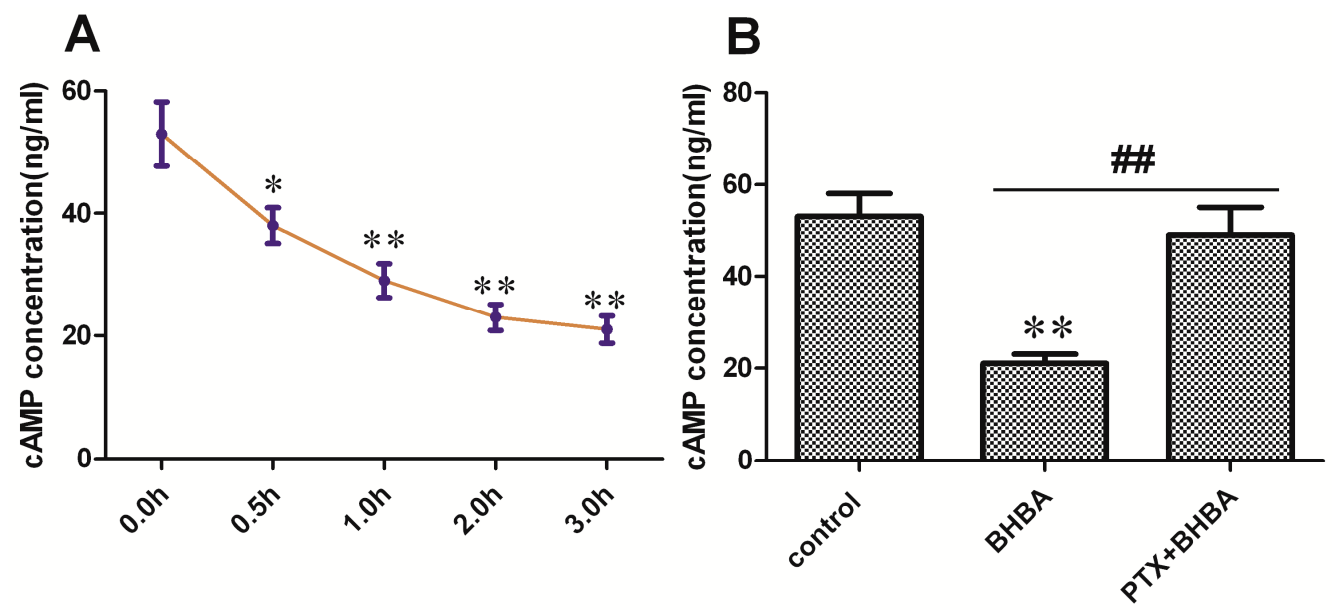

Figure 3. The effect of BHBA on intracellular cAMP levels in DCAPCs. (A) DCAPCs were treated with $2.5 \mathrm{mmol} / \mathrm{L}$ BHBA for $0,0.5,1.0,2.0$, and $3.0 \mathrm{~h}$; (B) The cells were also treated with or without prior PTX incubation for $2 \mathrm{~h}$ and then stimulated with $2.5 \mathrm{mmol} / \mathrm{L}$ BHBA for $3 \mathrm{~h}$. * indicates $p<0.05 v s$. the control group, ** indicates $p<0.01 v s$. the control group, \#\# indicates $p<0.01 v s$. the prior PTX incubation group. 


\subsection{Effect of BHBA on PKA Activity}

The PKA activity was lower in the BHBA-treated groups than in the control group, and the inhibiting effect of BHBA was blocked by PTX (Figure 4). Thus, BHBA can inhibit PKA activity by decreasing the intracellular cAMP concentration in DCAPCs.

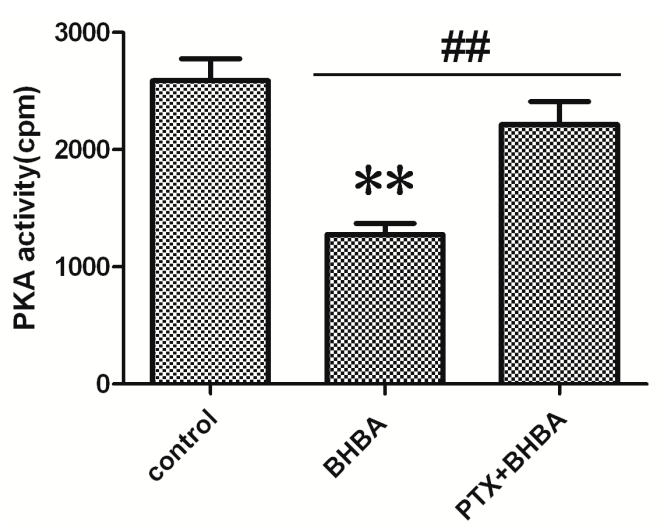

Figure 4. Effect of The effect of BHBA on the activity of PKA in DCAPCs. ** indicates $p<0.01$ vs. the control group, ${ }^{\#}$ indicates $p<0.01$ vs. the prior PTX incubation group.

\subsection{Effect of BHBA on CREB Phosphorylation}

The phosphorylation levels of CREB (Figure 5) were markedly lower in the BHBA-treated group than in the control group (Figure 5A; $p<0.01$ ), and the phosphorylation levels of CREB were markedly higher with prior PTX treatment (Figure 5B; $p<0.01$ ). Taken together, these findings suggest that BHBA inhibit the cAMP/PKA/CREB signaling pathway to modulate $G H$ and $P R L$ gene transcription in DCAPCs.
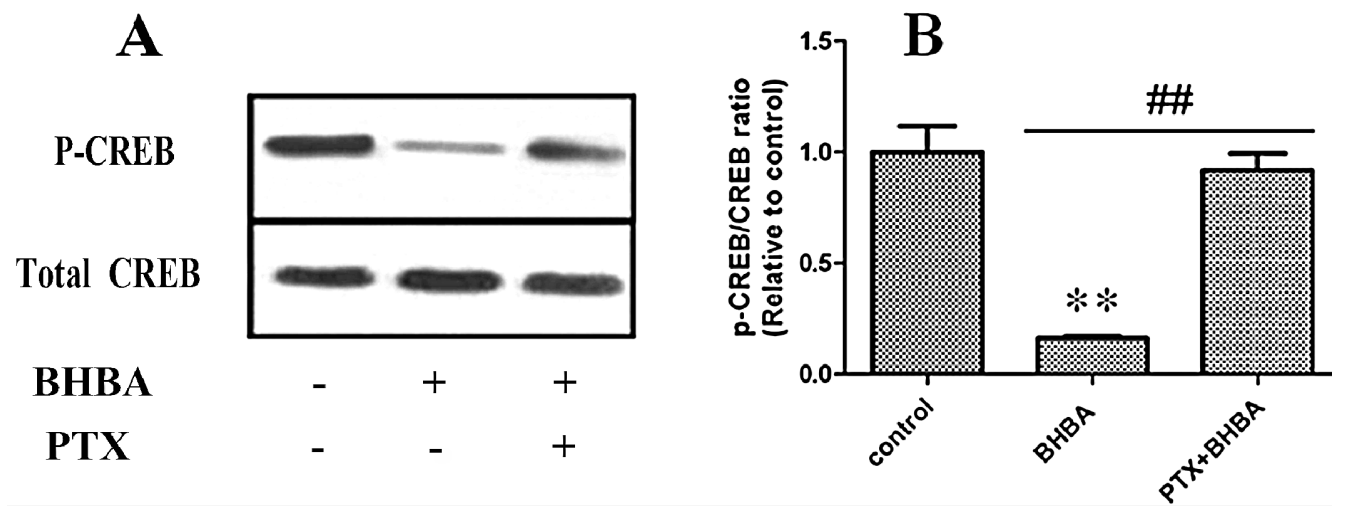

Figure 5. The effect of BHBA on CREB phosphorylation in DCAPCs. (A) The Western blotting results of $\mathrm{p}-\mathrm{CREB}$ and CREB; (B) The phosphorylation level of CREB. ** indicates $p<0.01$ vs. the control group, ${ }^{\#}$ indicates $p<0.01$ vs. the prior PTX incubation group.

\subsection{Effect of BHBA on the mRNA Levels of GPR109A and MCT1 in DCAPCs}

GPR109A is the functional receptor for BHBA, and the uptake of BHBA into cells may be mediated by the monocarboxylate transporter 1 (MCT1) system. Thus, this study examined the effect of BHBA on 
the mRNA levels of GPR109A and MCT1 in DCAPCs. GPR109A mRNA levels exhibited no obvious change in the BHBA-treated groups when compared with the control group (Figure 6A; $p>0.05$ ). MCT1 mRNA levels were significantly increased after BHBA treatment for 3, 6 and $12 \mathrm{~h}$ (Figure $6 \mathrm{~B} ; p<0.05$ ).
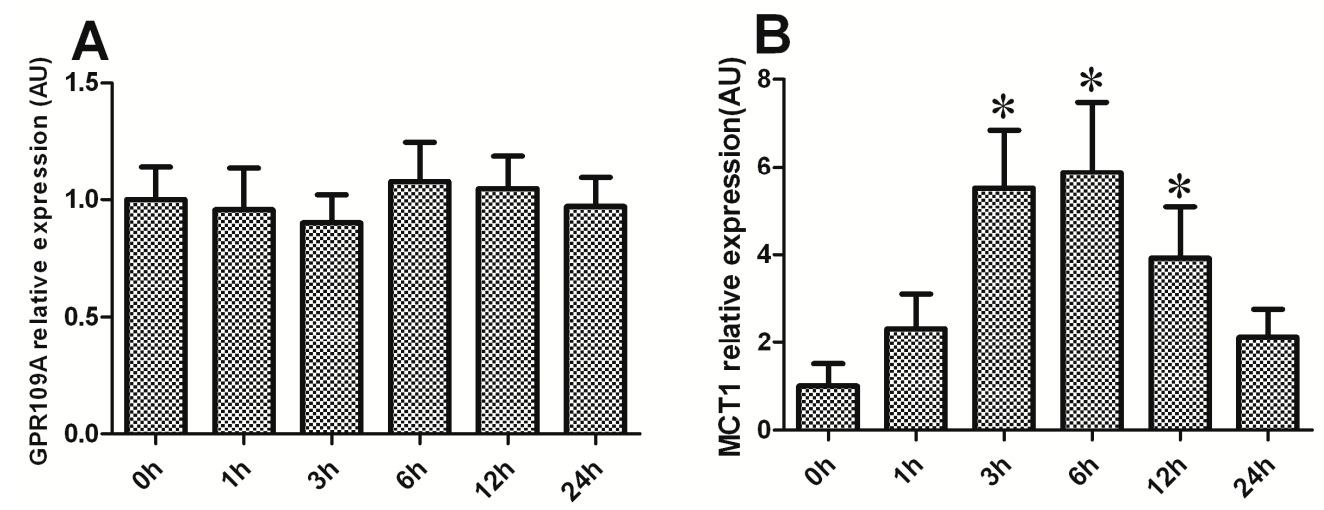

Figure 6. The effect of BHBA on the mRNA levels of GPR109A and MCT1 in DCAPCs. (A) The effect of BHBA on the mRNA levels of GPR109A in DCAPCs; (B) The effect of BHBA on the mRNA levels of MCT1 in DCAPCs. * indicates $p<0.05 v s$. the control group.

\subsection{The Role of AMPK- $\alpha$ in BHBA-Regulated GH and PRL Transcription and Secretion}

The activity of AMPK- $\alpha$ displayed no obvious change in DCAPCs cultured in the low-glucose and non-glucose groups when compared with the high-glucose group (Figure 7A; $p>0.05$ ). Incubation with $2.5 \mathrm{mmol} / \mathrm{L}$ BHBA resulted in a notable increase of AMPK- $\alpha$ activity in DCAPCs cultured in the low-glucose and non-glucose conditions when compared with the high-glucose group (Figure 7A). AMPK- $\alpha$ activity was markedly decreased by prior BML-275 treatment (Figure $7 \mathrm{~B} ; p<0.01$ ). For the DCAPCs cultured in the low-glucose and non-glucose conditions, incubation with $2.5 \mathrm{mmol} / \mathrm{L} \mathrm{BHBA}$ had no obvious effect on $G H$ and $P R L$ expression compared with the high-glucose group (Figure 7C; $p>0.05)$. In contrast, the secretion levels of GH and PRL were notably decreased following BHBA treatment in DCAPCs cultured in the low-glucose and non-glucose conditions compared with the high-glucose group (Figure 7D). In addition, the trend toward decreasing secretion levels of GH and PRL was attenuated by prior BML-275 treatment (Figure 7E,F). These results suggest that the intracellular uptake of BHBA mediated by MCT1 may trigger AMPK signaling and result in decreases in $G H$ and $P R L$ mRNA translation.
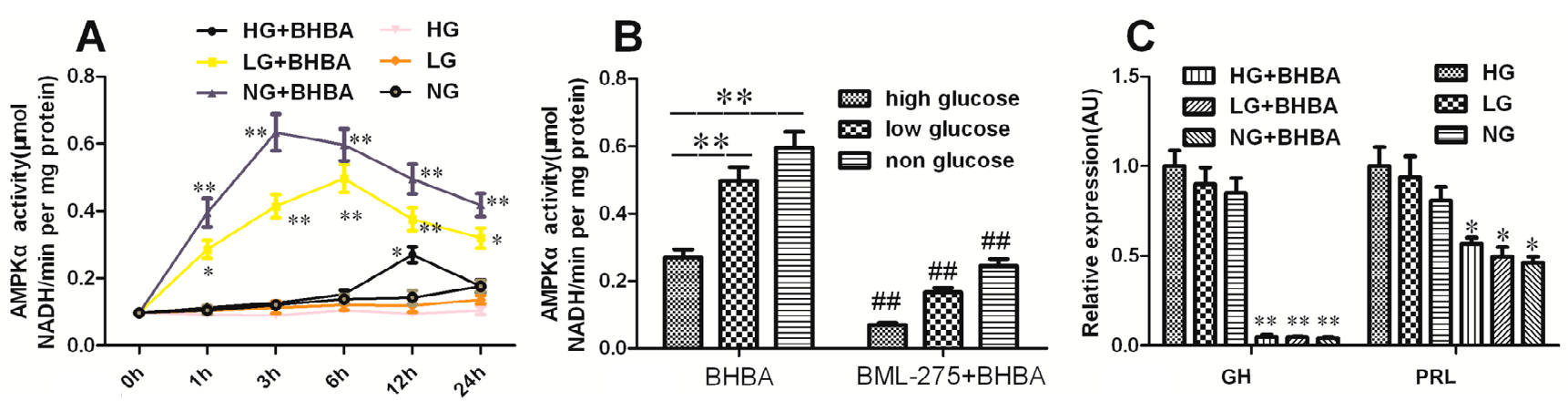

Figure 7. Cont. 

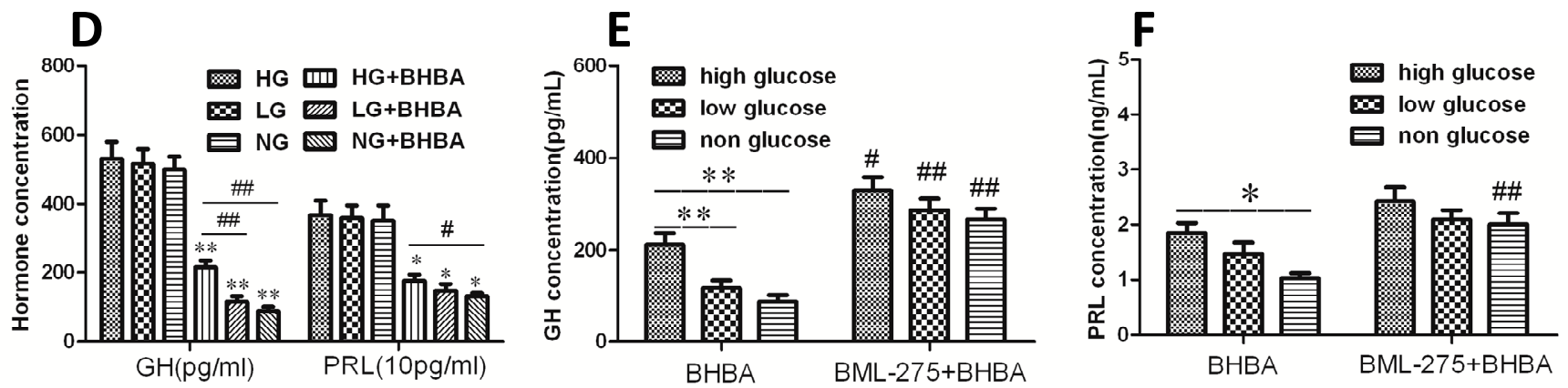

Figure 7. The role of AMPK- $\alpha$ in BHBA-regulated GH and PRL transcription and secretion in DCAPCs. (A) The effect of BHBA treatment on the activity of AMPK $\alpha$ in DCAPCs; (B) The effect of BML-275 treatment on the activity of AMPK $\alpha$ induced by BHBA in DCAPCs; (C) The effect of BHBA treatment on the gene expression of $G H$ and PRL in DCAPCs; (D) The effect of BHBA treatment on the secretion level of GH and PRL in DCAPCs; (E) The effect of BML-275 treatment on GH secretion inhibited by BHBA in DCAPCs; (F) The effect of BML-275 treatment on PRL secretion inhibited by BHBA in DCAPCs. HG indicates high glucose $(25.0 \mathrm{mM})$, LG indicates low glucose $(5.5 \mathrm{mM})$, and $\mathrm{NG}$ indicates non-glucose $(0.0 \mathrm{mM})$, respectively. ${ }^{*}$ indicates $p<0.05$ vs. the control group, ** indicates $p<0.01 v s$. the control group, ${ }^{*}$ indicates $p<0.05 v s$. the prior PTX incubation group, ${ }^{\#}$ indicates $p<0.01$ vs. the prior PTX incubation group.

\section{Discussion}

Growth hormone $(\mathrm{GH})$ is a polypeptide hormone synthesized and secreted by the anterior pituitary gland. In lactating cows, GH induces the proliferation of mammary parenchyma and the growth of epithelial cells and increases cell renewal in the mammary gland [11]. Moreover, GH increases milk protein gene expression in bovine mammary explants and mammary epithelial cells $[12,13]$. PRL is a polypeptide hormone that is synthesized in and secreted from lactotrophs of the anterior pituitary gland [14]. PRL also play a key role in regulating mammary gland development and lactation [15]. Because of their integral regulatory role in growth, metabolism, and lactation, the factors and mechanisms affecting GH and PRL synthesis and release have been emphasized in endocrinology research.

BHBA has been identified as an endogenous ligand of GPR109A [5]. Plaisance et al. found that BHBA stimulates adiponectin secretion through its action on the GPR109A receptor [16]. GPR109A is a seven-transmembrane $G$ protein-coupled receptor of the $G_{\alpha i}$ family that, in humans and mice, is expressed mainly in white adipocytes and immune cells such as monocytes and neutrophils.

In adipocytes, GPR109A activation results in the Gai/o protein-mediated inhibition of adenylate cyclase, leading to a decreased cAMP response [17]. The suppression of cAMP has also been reported in GPR109A-transfected CHO-K1, 293EBNA, and HEK293 cells, which is ascribed at least in part to the inhibition of adenylate cyclase mediated by GPR109A [17-19]. In this study, we investigated whether BHBA mediate bovine $G H$ and $P R L$ gene transcription via the $G$ protein signaling pathway. Our results demonstrate that BHBA can decrease intracellular cAMP concentration, PKA activity, and phosphorylation levels of CREB, by activating the $\mathrm{G}_{\alpha i}$ subunit in DCAPCs. In the nucleus, phosphorylated CREB could either directly modulate $G H$ gene transcription levels or indirectly activate Pit-1 to trigger the transcription 
of the $G H$ and $P R L$ genes [20-22]. In the present study, BHBA significantly down-regulated the phosphorylation levels of CREB. Thus, the expression and secretion of GH and PRL in DCAPCs were significantly decreased. PTX catalyzes the ADP-ribosylation of the $\alpha$ subunits of the heterotrimeric $\mathrm{G}_{\mathrm{i} / \mathrm{o}}$ protein family, thereby preventing the $\mathrm{G}$ proteins from interacting with their cognate GPCRs. This modification of the Gai/o proteins results in the enhanced accumulation of cAMP, which is one of the mechanisms by which PTX induces the various biological effects in its host cells. Therefore, the expression and secretion levels of GH and PRL were significantly higher following prior PTX incubation. Based on these observations, we conclude that BHBA can inhibit $G H$ and PRL gene transcription and secretion via the $\mathrm{cAMP} / \mathrm{PKA} / \mathrm{CREB}$ signaling pathway.

GPR109A belongs to a family of three GPCRs that share a significant sequence homology and whose known cognate ligands are metabolites of BHBA. GPR109A has generated increasing interest since its discovery as the receptor for niacin a decade ago. Taggart et al. demonstrated that the ketone body BHBA is a ligand for the receptor at physiologic concentrations [5]. Butyrate was also able to activate the receptor. As GPR109A's primary pharmacological ligand in clinical use, niacin has been used for over 50 years in the treatment of cardiovascular disease, mainly due to its favorable effects on plasma lipoproteins [23]. In addition, the activation of GPR109A with agonists also mediates anti-inflammatory effects [23], tumor-suppressive effects [24], and immunoregulation [25]. Titgemeyer et al. first reported that cattle contain GPR109A in fat, liver, muscle, and brain tissue [6]. The authors suggested that the ability of BHBA to bind and activate GPR109A makes the unusual distribution of GPR109A in cattle especially intriguing. In this study, GPR109A mRNA was observed in bovine anterior pituitary gland, which suggests that BHBA may act directly at the level of the pituitary.

Cows can generate BHBA both in the liver by ketogenesis during a state of negative energy balance and via the oxidation of butyrate exclusive in ruminal epithelial cells [4]. In bovines, clinical ketosis is defined as increases in serum BHBA and NEFA concentration is association with a decrease in serum glucose concentrations [26]. Additionally, Zarrin et al. reported that plasma glucose concentrations decreased dramatically in response to an infusion of BHBA in lactating cows [27]. AMPK is activated under conditions that deplete cellular ATP and elevate AMP levels, such as occur during glucose deprivation and hypoxia [10]. In addition, the incubation of GT1-7 cells with BHBA in the $5.5 \mathrm{mM}$ glucose medium were shown to modulate AMPK- $\alpha$ phosphorylation in GT1-7 cells [8]. Therefore, in the present study, we investigated whether BHBA regulates bovine pituitary function through the activation of AMPK- $\alpha$ signaling in cows during period of low blood glucose concentration. As expected, the levels of GH and PRL secretion were notably decreased after BHBA treatment in DCAPCs cultured in the low-glucose and non-glucose conditions in comparison with the high-glucose group. In addition, the observed trend of a decrease in levels of GH and PRL secretion was attenuated by prior BML-275 treatment. These results suggest that BHBA can trigger AMPK signaling and regulate bovine pituitary function.

AMPK is a heterotrimeric serine/threonine kinase that is involved in the maintenance of energy homeostasis and recovery from metabolic stress both at the cellular and whole-body level. Furthermore, AMPK has been found in all tissues examined thus far, and a number of its downstream targets have been identified. Several known AMPK substrates include key enzymes involved in lipid and glucose metabolism (e.g., Acetyl-coenzyme A carboxylase 1/2, ACC1/2; 3-hydroxy-3-methyl glutaryl coenzyme A reductase, HMG CoA; Insulin receptor substrate 1, IRS1), transcriptional components (e.g., CREB 
binding protein, CBP; Peroxisome proliferator-activated receptor $\gamma, \operatorname{PPAR} \gamma$ ), and components of the mammalian target of rapamycin (mTOR) signaling pathway (e.g., tuberous sclerosis complex, TSC1-TSC2) [28-30]. The CBP acts as a cofactor for the Pit-1-dependent activation of the hGH promoter by the GHRH signaling pathway and PKA [31]. Moreover, CBP acts by binding to phosphorylated CREB and activating gene expression [32]. The phosphorylated CREB may increase the levels of phosphorylated CBP or CBP complex, which interact with Pit-1 and result in the transcriptional activation of the $G H$ and $P R L$ genes. However, incubation with BHBA had no obvious effect on $G H$ and $P R L$ gene expression in DCAPCs cultured in the low-glucose and non-glucose groups. In contrast, GH and PRL secretion levels were notably decreased after BHBA treatment in DCAPCs cultured in the low-glucose and non-glucose groups. In mammals, the TSC1/TSC2-complex integrates environmental signals such as energy status and growth factors into mTOR signaling. In the case of stress (e.g., DNA damage, hypoxia) or low energy availability, the TSC1/TSC2-complex is activated and regulates protein synthesis down [33]. The trend toward decreasing secretion levels of GH and PRL was attenuated by prior BML-275 treatment. Thus, we hypothesize that BHBA may trigger AMPK signaling, and result in the phosphorylation of TSC1-TSC2, thereby leading to a reduction in $G H$ and $P R L$ mRNA translation via mTOR signaling. We found that the mRNA levels of $M C T 1$ were significantly increased after BHBA treatment. Based on these observations, we conclude that the intracellular uptake of BHBA mediated by MCT1 can trigger AMPK signaling and result in decrease in GH and PRL mRNA translation.

In summary, the results of this study indicate that BHBA, acting as a signaling molecule, significantly decreases $G H$ and PRL gene transcription in DCAPCs via the putative mechanism illustrated in Figure 8. Specifically, BHBA binds to GPR109A and leads to the dissociation of the heterotrimeric $\mathrm{G}$ protein complex into $\mathrm{G}_{\alpha \mathrm{i}}$ and $\beta \gamma$ subunits. Next, the exchange of GTP from GDP results in the activation of the $\mathrm{G}_{\alpha i}$, subunits, thereby inhibiting adenylyl cyclase activity. The inactivation adenylyl cyclase leads to a decrease in intracellular cAMP levels and a subsequent reduction in PKA activity. The inhibition of PKA activity inhibits CREB phosphorylation, which leads to a decrease in bovine GH and Pit-1 gene transcription. The subsequent change in Pit-1 content results in the inhibition of transcription of the bovine $G H$ and $P R L$ genes. Consequently, BHBA inhibits bovine $G H$ and $P R L$ gene transcription and secretion in DCAPCs. The A-protomer of PTX penetrates into the host cells and results

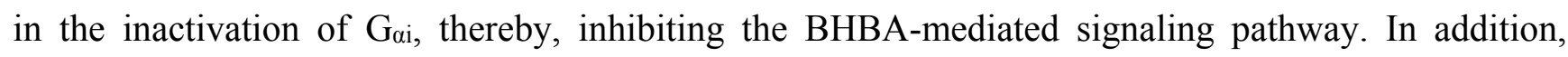
intracellular BHBA uptake mediated by MCT1 may trigger AMPK signaling and result in the phosphorylation of TSC1-TSC2, leading to a decrease in $G H$ and PRL mRNA translation via mTOR signaling. mTOR integrates growth signals from diverse mechanisms that sense nutrient availability and as part of the response regulating cell survival [34]. We also found that cell survival was notably decreased after BHBA treatment in DCAPCs cultured under low-glucose and non-glucose condition when compared with the high-glucose group (our unpublished data). Therefore, BHBA may also decrease cell survival in DCAPCs cultured in condition of low and no glucose group. This study identifies a biochemical mechanism for the regulatory action of BHBA on $G H$ and PRL gene transcription, translation, and secretion in DCAPCs, which may be one of the factors that regulate pituitary function during the transition period in dairy cows. Future studies are needed to clarify the specific role and mechanism of BHBA in regulating the survival of DCAPCs. 


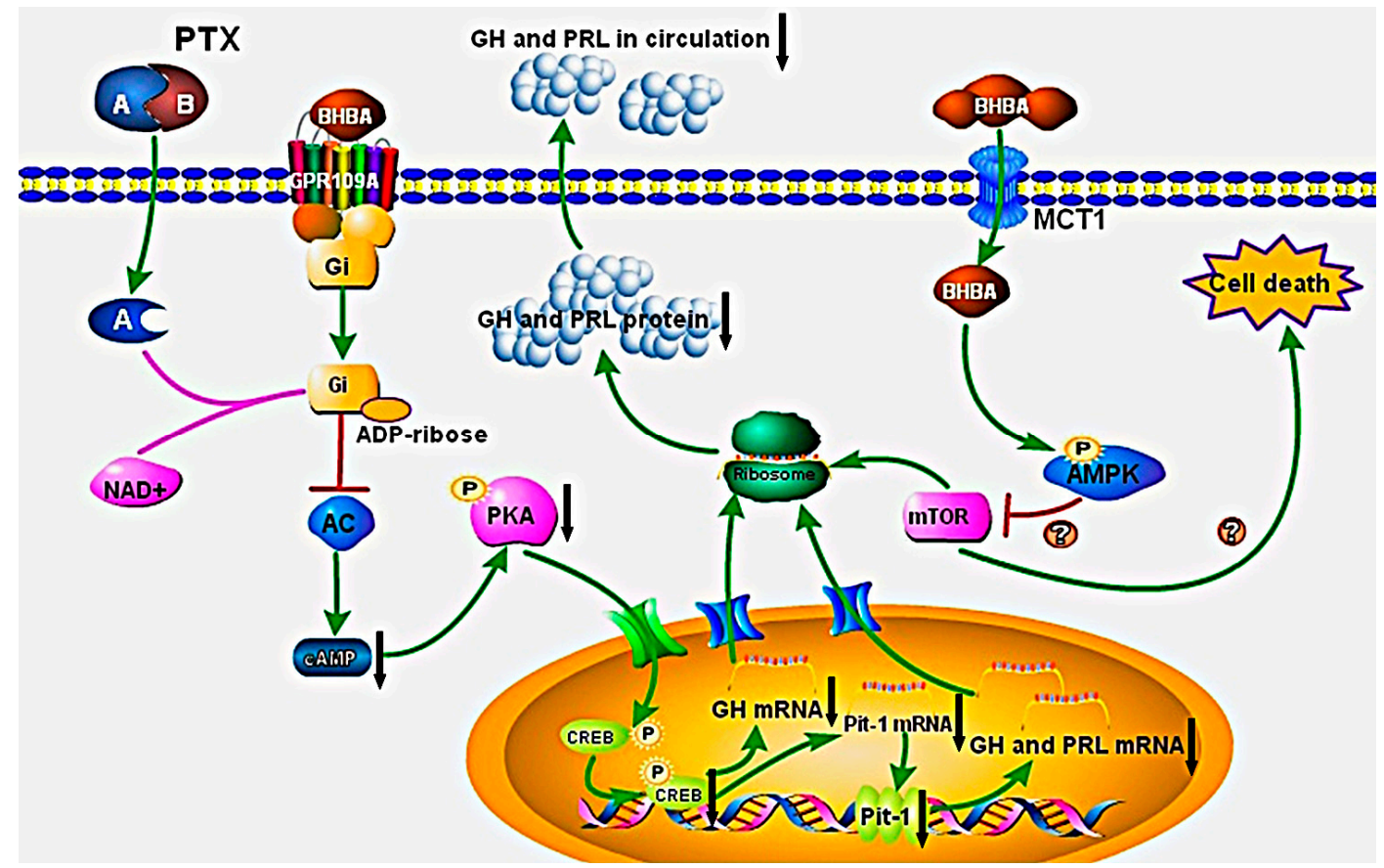

Figure 8. BHBA mediates $\mathrm{GH}$ and $\mathrm{PRL}$ gene transcription, translation, and secretion in DCAPCs via the cAMP/PKA/CREB and AMPK signaling pathways. BHBA binds to GPCR and leads to the dissociation of the heterotrimeric $G$ protein complex into $G_{\alpha i}$ and $G_{\beta \gamma}$ subunits. The exchange of GTP from GDP results in the activation of $\mathrm{G}_{\alpha i}$, thereby inhibiting adenylyl cyclase (AC) activity. This process results in a decrease in intracellular cAMP levels and a subsequent reduction in PKA activity. The inhibition of PKA activity inhibits CREB phosphorylation, thereby decreasing GH and PRL gene transcription, translation, and secretion directly or indirectly. The A-protomer of PTX penetrates into the host cells and results in the inactivation of $\mathrm{G}_{\alpha \mathrm{i}}$, which subsequently inhibits the BHBA-mediated signaling pathway. In addition, intracellular BHBA uptake mediated by MCT1 may trigger AMPK signaling and result in the phosphorylation of TSC1-TSC2, leading to a decrease in $G H$ and $P R L$ mRNA translation via mTOR signaling.

\section{Materials and Methods}

\subsection{Isolation and Culture of DCAPCs}

The cells were isolated and cultured by the enzymatic digestion method as previously described [35]. In brief, three Holstein cows (at the fifth lactational stage) anterior pituitary glands were diced into small pieces of less than $1 \mathrm{~mm}^{3}$, and incubated in Hanks' balanced salt solution without calcium and magnesium (CMF-HBSS) containing 0.3\% I type collagenase, $0.1 \%$ hyaluronidase and 0.1\% DNase (Sigma, Shanghai, China) at $37^{\circ} \mathrm{C}$ for $2 \mathrm{~h}$. The dispersed cells were washed three times with HBSS and resuspended in Dulbecco's modified Eagle's medium (DMEM; Gibco, $25.0 \mathrm{mM}$ glucose, Gibco, Carlsbad, CA, USA) supplemented with $10 \%$ fetal bovine serum (FBS; Gibco) at a seeding density of $1 \times 10^{3}$ cells $/ \mathrm{mL}$. Then, the cells were seeded into a $75 \mathrm{~cm}^{2}$ culture flask (Corning, Tewksbury, MA, USA) and incubated at $37{ }^{\circ} \mathrm{C}$ in a humidified atmosphere containing $5 \% \mathrm{CO}_{2}$. After $6 \mathrm{~d}$ in culture, the cells were treated with $0.5 \%$ trypsin (Sigma) and $0.02 \%$ EDTA in CMF-PBS and then seeded into 
6-cm cell culture dishes (Corning). The anterior pituitary cells at the second passage were used to perform the experiment.

\subsection{RNA Extraction, Reverse Transcriptase, and Quantitative Real-Time PCR Analysis}

The time course of the experiments consisted of the following stages: DCAPCs were grown in 6-well plates $\left(5 \times 10^{5}\right.$ cells $)$, subsequently serum starved for $24 \mathrm{~h}$, and then stimulated with $1.0 \mathrm{mmol} / \mathrm{L}$ DL- $\beta$-hydroxybutyric acid sodium salt (BHBA; Sigma) for $0,1,3,6,12$, and $24 \mathrm{~h}$. For the dose-response experiments, DCAPCs were stimulated with $0,0.1,0.5,1.0,2.5$, and $5.0 \mathrm{mmol} / \mathrm{L} \mathrm{BHBA}$ for $24 \mathrm{~h}$. PTX is the ADP-ribosylating toxin produced by the whooping cough-causing bacterium Bordetella pertussis. ADP-ribosylation of the $\alpha$ subunit of heterotrimeric $\mathrm{G}_{\alpha i}$ proteins locks the $\alpha$ subunits into an inactive state, thus preventing them from inhibiting adenylyl cyclase [36]. This method is widely applied as a tool in biochemical and pharmacological studies to investigate of signaling pathways involving heterotrimeric G proteins [37]. DCAPCs were also treated with or without prior PTX (Sigma) incubation $(100 \mathrm{ng} / \mathrm{L})$ for $2 \mathrm{~h}$ and then stimulated with $1.0 \mathrm{mmol} / \mathrm{L}$ BHBA for $24 \mathrm{~h}$. Each treatment concentration of BHBA or PTX was replicated 12 times. The cell supernatants and cells were collected for hormone analysis and RNA extraction, respectively. Total RNA was isolated from cells with TRIzol reagent (Sigma) according to the manufacturer's instructions and treated with DNase I (Takara, Kyoto, Japan) to prevent genomic DNA contamination. Total RNA concentration and purity were determined using a spectrophotometer (Bio-Rad, Hercules, CA, USA). Only samples with an optical density ratio at $260 / 280 \mathrm{~nm}$ in the range of 1.8-2.2 were used for further analysis. Total RNA integrity was checked by electrophoresis on an agarose gel. Total RNA samples were reverse transcribed using a reverse transcription kit (Takara) in accordance with to the manufacturer's instructions. The expression levels of various genes were evaluated by quantitative polymerase chain reaction (qRT-PCR) analysis using the SYBR Green QuantiTect RT-PCR Kit (Roche, South San Francisco, CA, USA), performed in triplicate for each sample. The relative expression levels of bovine GH, PRL, Pit-1, GPR109A and MCT1 (BHBA uptake via $M C T 1$ ) genes were calculated relative to $G A P D H$ (the normalizer) using the comparative cycle threshold method. The primer sequences for the tested genes are shown in Table 1 as previously described $[6,38,39]$.

Table 1. The primer sequences of bovine GAPDH, GH, PRL, Pit-1, GPR109A and MCT1.

\begin{tabular}{ccc}
\hline Gene & Sequences & Length (bp) \\
\hline \multirow{2}{*}{ GAPDH } & (F) 5'-TGCCCAGAATATCATCCC-3' & 134 \\
& (R) 5'-AGGTCAGATCCACAACAG-3' & \\
GH & (F) 5'-AGATCCTCAAGCAGACCTA-3' & 121 \\
& (R) 5'-AGGTACGTCTCCGTCTTA-3' & \\
PRL & (F) 5'-TATGAAAGGAGCCCCAGATG-3' & 137 \\
& (R) 5'-CACACAGGGTAGGGCTCAGT-3' & \\
\multirow{2}{*}{ Pit-1 } & (F) 5'-TTCTGCAACTCTGCCTCTGA-3' & 148 \\
\multirow{2}{*}{ GPR109A } & (R) 5'-CCATAGGTCGATGACTGGT-3' & \\
& (F) 5'-ACATCACCCTCAGCTTCACC-3' & 146 \\
\multirow{2}{*}{$M C T 1$} & (R) 5'-GCGGTTGTTATCCGACTCAT-3' & \\
& (F) 5'-GGAGTCATTGGAGGTCTTGG-3' & 137 \\
\hline
\end{tabular}




\subsection{Measurement of GH and PRL Concentration in the Supernatant}

GH and PRL in cell supernatants were evaluated with the corresponding ELISA kits according to the instructions of the manufacturer (Chemicon International, Millipore, Billerica, MA, USA).

\subsection{Measurement of Intracellular cAMP Concentration}

DCAPCs were grown in 6-well plates $\left(5 \times 10^{5}\right.$ cells $)$, serum starved for $24 \mathrm{~h}$, and then stimulated with $1.0 \mathrm{mmol} / \mathrm{L}$ BHBA for $0.5,1,2$, and $3 \mathrm{~h}$. The intracellular cAMP was extracted from the cells and the concentration was assayed with a microplate reader (Bio-Rad, Hercules, CA, USA) using a cAMP assay kit (R\&D Systems, Minneapolis, MN, USA) according to the manufacturer's instructions. DCAPCs were also treated with or without prior PTX incubation $(100 \mathrm{ng} / \mathrm{L})$ for $2 \mathrm{~h}$ and then stimulated with $1.0 \mathrm{mmol} / \mathrm{L}$ BHBA for $3 \mathrm{~h}$. Each treatment concentration of BHBA or PTX was replicated 12 times. The assay is based on the competition between unlabeled cAMP and a fixed quantity of horseradish peroxidase (HRP)-labeled cAMP for the limited number of binding sites on a cAMP specific antibody.

\subsection{Measurement of PKA Activity}

cAMP works by activating protein kinase A (PKA), which leads to the phosphorylation of cAMP response element binding protein (CREB). Thus, the PKA activity and phosphorylation state of CREB were determined in this study. DCAPCs were cultured in the presence or absence of BHBA $(1.0 \mathrm{mmol} / \mathrm{L})$ in serum-free medium for $3 \mathrm{~h}$. The cells were also treated with prior PTX incubation $(100 \mathrm{ng} / \mathrm{L})$ for $2 \mathrm{~h}$ compared with groups without prior PTX incubation. Each treatment concentration of BHBA or PTX was replicated 12 times. The activity of PKA was determined by a radioactive method from a PKA assay kit according to the manufacturer's instructions (Millipore).

\subsection{Western Blotting Analysis of Phosphorylated CREB}

Nuclear proteins were extracted using a nuclear protein extraction kit according to the manufacturer's instructions (Beyotime Co., Nantong, China). Protein concentrations were determined with a bicinchoninic acid protein assay kit (Beyotime). Aliquots of cell lysates containing $20 \mu \mathrm{g}$ protein were separated in $10 \%$ polyacrylamide gels and electrophoretically transferred to PVDF membranes (Millipore) using a Bio-Rad criterion blotter. Membranes were blocked in 5\% non-fat dried milk in TBST at room temperature for $1 \mathrm{~h}$ and then incubated with either anti-phospho-CREB (1:3000 dilution; Millipore) or anti-CREB (1:2000 dilution; Millipore) at $4{ }^{\circ} \mathrm{C}$ overnight. The blots were then washed and incubated with horseradish peroxidase-labeled secondary antibodies (Sigma) at $37{ }^{\circ} \mathrm{C}$ for $1 \mathrm{~h}$. Immunoreactive bands were detected with enhanced chemiluminescence Western blotting detection reagents (Beyotime). The blots were exposed to X-ray film for radiography of the bands and were digitally detected and measured using a LAS3000 Bioimage Analyzer (Fuji Photo Film, Tokyo, Japan).

\subsection{Measurement of AMPK- $\alpha$ Activity}

DCAPCs were grown in 6-well plates $\left(5 \times 10^{5}\right.$ cells $)$ and maintained in high- $(25.0 \mathrm{mM})$, low- $(5.5 \mathrm{mM})$, and non- $(0.0 \mathrm{mM})$ glucose DMEM, serum starved for $24 \mathrm{~h}$, and then stimulated with $1.0 \mathrm{mmol} / \mathrm{L} \mathrm{BHBA}$ 
for $0,1,3,6,12$, and $24 \mathrm{~h}$. BML-275 is an AMPK- $\alpha$ inhibitor that inhibits AMPK- $\alpha$ phosphorylation at threonine-172. The cells were also treated with prior BML-275 incubation $(10 \mu \mathrm{mol} / \mathrm{L})$ for $2 \mathrm{~h}$ compared with groups without prior BML-275 incubation. Each treatment concentration of BHBA or BML-275 was replicated 12 times. Protein was extracted by cell lysis method described by Li et al. [40]. The enzyme activity of AMPK- $\alpha$ was detected using a biochemical kit via spectrophotometry (Shanghai Bluegene Biotech Co., Ltd., Shanghai, China) according to the manufacturer's instructions.

\subsection{Statistical Analyses}

Results were expressed as means \pm SD. Data were analyzed by using statistical software package SPSS 12.0 (SPSS Inc., Chicago, IL, USA). Groups were compared by one-way analysis of variance (ANOVA) followed by the least significant difference test. A $p$ value of less than 0.05 was considered statistically significant, and values less than 0.01 were considered markedly significant.

\section{Acknowledgments}

This work was founded by National Key Basic Research Program of China (project No. 2011CB100805), National Natural Science Foundation of China (Grant No. 31402157 and 31472249), Jilin Scientific and Technological Development Program (project No. 20130206036 NY) and Key Laboratory of Veterinary Medicine of Heilongjiang Bayi Agricultural University in Heilongjiang Provincial University.

\section{Author Contributions}

Shou-Peng Fu, Wei Wang, Bing-Run Liu, Huan-Min Yang, Hong Ji, Zhan-Qing Yang and Bin Guo performed the experiments; Jian-Fa Wang and Ju-Xiong Liu designed the study; Shou-Peng Fu and Jian-Fa Wang wrote the manuscript.

\section{Conflicts of Interest}

The authors declare no conflict of interest.

\section{References}

1. Shi, X.; Li, D.; Deng, Q.; Li, Y.; Sun, G.; Yuan, X.; Song, Y.; Wang, Z.; Li, X.; Li, X.; et al. NEFAs activate the oxidative stress-mediated NF- $\mathrm{KB}$ signaling pathway to induce inflammatory response in calf hepatocytes. J. Steroid Biochem. Mol. Biol. 2015, 145, 103-112.

2. Ooi, G.T.; Tawadros, N.; Escalona, R.M. Pituitary cell lines and their endocrine applications. Mol. Cell Biol. 2004, 228, 1-21.

3. Meier, S.; Gore, P.J.; Barnett, C.M.; Cursons, R.T.; Phipps, D.E.; Watkins, K.A.; Verkerk, G.A. Metabolic adaptations associated with irreversible glucose loss are different to those observed during under-nutrition. Domest. Anim. Endocrinol. 2008, 34, 269-277.

4. Laeger, T.; Metges, C.C.; Kuhla, B. Role of beta-hydroxybutyric acid in the central regulation of energy balance. Appetite 2010, 54, 450-455. 
5. Taggart, A.K.; Kero, J.; Gan, X.; Cai, T.Q.; Cheng, K.; Ippolito, M.; Ren, N.; Kaplan, R.; Wu, K.; $\mathrm{Wu}, \mathrm{T} . J . ;$ et al. (D)- $\beta$-Hydroxybutyrate inhibits adipocyte lipolysis via the nicotinic acid receptor PUMA-G. J. Biol. Chem. 2005, 280, 26649-26652.

6. Titgemeyer, E.C.; Mamedova, L.K.; Spivey, K.S.; Farney, J.K.; Bradford, B.J. An unusual distribution of the niacin receptor in cattle. J. Dairy Sci. 2011, 94, 2177-2187.

7. Wang, J.F.; Fu, S.P.; Li, S.N.; Hu, Z.M.; Xue, W.J.; Li, Z.Q.; Huang, B.X.; Lv, Q.K.; Liu, J.X.; Wang, W. Short-chain fatty acids inhibit growth hormone and prolactin gene transcription via cAMP/PKA/CREB signaling pathway in dairy cow anterior pituitary cells. Int. J. Mol. Sci. 2013, 14, 21474-21488.

8. Laeger, T.; Pöhland, R.; Metges, C.C.; Kuhla, B. The ketone body $\beta$-hydroxybutyric acid influences agouti-related peptide expression via AMP-activated protein kinase in hypothalamic GT1-7 cells. J. Endocrinol. 2012, 213, 193-203.

9. Pelletier, A.; Coderre, L. Ketone bodies alter dinitrophenol-induced glucose uptake through AMPK inhibition and oxidative stress generation in adult cardiomyocytes. Am. J. Physiol. Endocrinol. Metab. 2007, 292, E1325-E1332.

10. Tulipano, G.; Giovannini, M.; Spinello, M.; Sibilia, V.; Giustina, A.; Cocchi, D. AMP-activated protein kinase regulates normal rat somatotroph cell function and growth of rat pituitary adenomatous cells. Pituitary 2011, 14, 242-252.

11. Capuco, A.V.; Wood, D.L.; Baldwin, R.; McLeod, K.; Paape, M.J. Mammary cell number, proliferation, and apoptosis during a bovine lactation: Relation to milk production and effect of Bst. J. Dairy Sci. 2001, 84, 2177-2187.

12. Yang, J.; Zhao, B.; Baracos, V.E.; Kennelly, J.J. Effects of bovine somatotropin on beta-casein mRNA levels in mammary tissue of lactating cows. J. Dairy Sci. 2005, 88, 2806-2812.

13. Yonekura, S.; Sakamoto, K.; Komatsu, T.; Hagino, A.; Katoh, K.; Obara, Y. Growth hormone and lactogenic hormones can reduce the leptin mRNA expression in bovine mammary epithelial cells. Domest. Anim. Endocrinol. 2006, 31, 88-96.

14. Freeman, M.E.; Kanyicska, B.; Lerant, A.; Nagy, G. Prolactin: Structure, function, and regulation of secretion. Physiol. Rev. 2000, 4, 1523-1631.

15. Trott, J.F.; Schennink, A.; Petrie, W.K.; Manjarin, R.; VanKlompenberg, M.K.; Hovey, R.C. Triennial Lactation Symposium: Prolactin: The multifaceted potentiator of mammary growth and function. J. Anim. Sci. 2012, 95, 1674-1686.

16. Plaisance, E.P.; Lukasova, M.; Offermanns, S.; Zhang, Y.; Cao, G.; Judd, R.L. Niacin stimulates adiponectin secretion through the GPR109A receptor. Am. J. Physiol. Endocrinol. Metab. 2009, 296, E549-E558.

17. Tunaru, S.; Kero, J.; Schaub, A.; Wufka, C.; Blaukat, A.; Pfeffer, K.; Offermanns, S. Puma-G and HM74 are receptors for nicotinic acid and mediate its anti-lipolytic effect. Nat. Med. 2003, 9, $352-355$.

18. Soga, T.; Kamohara, M.; Takasaki, J.; Matsumoto, S.; Saito, T.; Ohishi, T.; Hiyama, H.; Matsuo, A.; Matsushime, H.; Furuichi, K. Molecular identification of nicotinic acid receptor. Biochem. Biophys. Res. Commun. 2003, 303, 364-369. 
19. Zhang, Y.; Schmidt, R.J.; Foxworthy, P.; Emkey, R.; Oler, J.K.; Larg, T.H.; Wang, H.; Su, E.W.; Mosior, M.K.; Eacho, P.I.; et al. Niacin mediates lipolysis in adipose tissue through its G-protein coupled receptor HM74A. Biochem. Biophys. Res. Commun. 2005, 334, 729-732.

20. Nelson, C.; Albert, V.R.; Elsholtz, H.P.; Lu, L.I.; Rosenfeld, M.G. Activation of cell-specific expression of rat growth hormone and prolactin genes by a common transcription factor. Science 1988, 239, 1400-1405.

21. Sanchez-Pacheco, A.; Palomino, T.; Aranda, A. Negative regulation of expression of the pituitary-specific transcription factor GHF-1/Pit-1 by thyroid hormones through interference with promoter enhancer elements. Mol. Cell. Biol. 1995, 15, 6322-6330.

22. Melmed, S. The Pituitary, 3rd ed.; Academic Press: London, UK, 2011; pp. 83-166.

23. Chai, J.T.; Digby, J.E.; Choudhury, R.P. GPR109A and vascular inflammation. Curr. Atheroscler. Rep. 2013, 15, 325.

24. Singh, N.; Gurav, A.; Sivaprakasam, S.; Brady, E.; Padia, R.; Shi, H.; Thangaraju, M.; Prasad, P.D.; Manicassamy, S.; Munn, D.H.; et al. Activation of GPR109A, receptor for niacin and the commensal metabolite butyrate, suppresses colonic inflammation and carcinogenesis. Immunity 2014, 40, 128-139.

25. Kostylina, G.; Simon, D.; Fey, M.F.; Yousefi, S.; Simon, H.U. Neutrophil apoptosis mediated by nicotinic acid receptors (GPR109A). Cell Death Differ. 2008, 15, 134-142.

26. Dowden, D.R.; Jacobson, D.R. Inhibition of appetite in dairy cattle by certain intermediate metabolities. Nature 1960, 188, 148-149.

27. Zarrin, M.; de Matteis, L.; Vernay, M.C.; Wellnitz, O.; van Dorland, H.A.; Bruckmaier, R.M. Long-term elevation of $\beta$-hydroxybutyrate in dairy cows through infusion: Effects on feed intake, milk production, and metabolism. J. Dairy Sci. 2013, 96, 2960-2972.

28. Gwinn, D.M.; Shackelford, D.B.; Egan, D.F.; Mihaylova, M.M.; Mery, A.; Vasquez, D.S.; Turk, B.E.; Shaw, R.J. AMPK phosphorylation of raptor mediates a metabolic checkpoint. Mol. Cell 2008, 30, 214-226.

29. Yang, W.; Hong, Y.H.; Shen, X.Q.; Frankowski, C.; Camp, H.S.; Leff, T. Regulation of transcription by AMP-activated protein kinase: Phosphorylation of p300 blocks its interaction with nuclear receptors. J. Biol. Chem. 2001, 276, 38341-38344.

30. Li, X.; Li, Y.; Yang, W.; Xiao, C.; Fu, S.; Deng, Q.; Ding, H.; Wang, Z.; Liu, G.; Li, X. SREBP-1c overexpression induces triglycerides accumulation through increasing lipid synthesis and decreasing lipid oxidation and VLDL assembly in bovine hepatocytes. J. Steroid Biochem. Mol. Biol. 2014, 143, 174-182.

31. Cohen, L.E.; Hashimoto, Y.; Zanger, K.; Wondisford, F.; Radovick, S. CREB-independent regulation by $\mathrm{CBP}$ is a novel mechanism of human growth hormone gene expression. J. Clin. Investig. 1999, 104, 1123-1130.

32. Lundblad, J.R.; Kwok, R.P.S.; Laurance, M.E.; Harter, M.L.; Goodman, R.H. Adenoviral E1A-associated p300 as a functional homologue of the transcriptional co-activator CBP. Nature 1995, 374, 85-88.

33. Inoki, K.; Ouyang, H.; Zhu, T.; Lindvall, C.; Wang, Y.; Zhang, X.; Yang, Q.; Bennett, C.; Harada, Y.; Stankunas, K.; et al. AMP-activated protein kinase: Ancient energy gauge provides clues to modern understanding of metabolism. Cell Metab. 2005, 1, 15-25. 
34. Hung, C.M.; Garcia-Haro, L.; Sparks, C.A.; Guertin, D.A. mTOR-dependent cell survival mechanisms. Cold Spring Harb. Perspect. Biol. 2012, 4, a008771.

35. Wang, J.F.; Fu, S.P.; Li, S.N.; Yang, Z.Q.; Xue, W.J.; Li, Z.Q.; Wang, W.; Liu, J.X. Establishment and characterization of dairy cow growth hormone secreting anterior pituitary cell model. In Vitro Cell. Dev. Biol. Anim. 2014, 50, 103-110.

36. Bruckener, K.E.; el Baya, A.; Galla, H.J.; Schmidt, M.A. Permeabilization in a cerebral endothelial barrier model by pertussis toxin involves the PKC effector pathway and is abolished by elevated levels of cAMP. J. Cell Sci. 2003, 116, 1837-1846.

37. Mangmool, S.; Kurose, H. Gi/o protein-dependent and -independent actions of pertussis toxin (PTX). Toxins 2011, 3, 884-899.

38. Laporta, J.; Driver, A.; Khatib, H. Short communication: Expression and alternative splicing of POU1F1 pathway genes in preimplantation bovine embryos. J. Dairy Sci. 2011, 94, 4220-4223.

39. Kirat, D.; Kato, S. Monocarboxylate transporter 1 (MCT1) mediates transport of short-chain fatty acids in bovine caecum. Exp. Physiol. 2006, 91, 835-844.

40. Li, X.; Li, X.; Chen, H.; Lei, L.; Liu, J.; Guan, Y.; Liu, Z.; Zhang, L.; Yang, W.; Zhao, C.; et al. Non-esterified fatty acids activate the AMP-activated protein kinase signaling pathway to regulate lipid metabolism in bovine hepatocytes. Cell Biochem. Biophys. 2013, 67, 1157-1169.

(C) 2015 by the authors; licensee MDPI, Basel, Switzerland. This article is an open access article distributed under the terms and conditions of the Creative Commons Attribution license (http://creativecommons.org/licenses/by/4.0/). 